\title{
Correction to: The Effectiveness of Resisted Sled Training (RST) for Sprint Performance: A Systematic Review and Meta-analysis
}

\author{
Pedro E. Alcaraz ${ }^{1,2} \cdot$ Jorge Carlos-Vivas $^{1} \cdot$ Bruno O. Oponjuru $^{1} \cdot$ Alejandro Martínez-Rodríguez $^{3}$
}

Published online: 9 July 2018

(c) Springer International Publishing AG, part of Springer Nature 2018

Correction to: Sports Medicine

https://doi.org/10.1007/s40279-018-0947-8

Page 1: Abstract, Conclusions, sentence 4. The following sentence, which read:

Moreover, the intensity (load) is not a determinant of sprint performance improvement, but the recommended volume is $>160 \mathrm{~m}$ per session, and approximately $2680 \mathrm{~m}$ per week, with a training frequency of two to three times per week, for at least 6 weeks.

should read:

Moreover, the intensity (load) is not a determinant of sprint performance improvement, but the recommended volume is $>160 \mathrm{~m}$ per session, and approximately $2680 \mathrm{~m}$ per total training program, with a training frequency of two to three times per week, for at least 6 weeks.

Page 6: Section 3.3, paragraph 3, sentence 2. The following sentence, which read:

The original article can be found online at https://doi.org/10.1007/ s40279-018-0947-8.

Pedro E. Alcaraz

pedro.e.alcaraz@gmail.com

Alejandro Martínez-Rodríguez

amartinezrodriguez@ua.es

1 UCAM Research Center for High Performance Sport, Catholic University of Murcia, Murcia, Spain

2 Faculty of Sport Sciences, UCAM, Catholic University of Murcia, Murcia, Spain

3 Department of Analytical Chemistry, Nutrition and Food Sciences, Faculty of Sciences, University of Alicante, Alicante, Spain
A large ES was obtained for a frequency over twice a week (ES 1.85) [55], and moderate ESs were found for lower loads (ES 0.61) [39, 55-57, 59-65], > 6-week training periods (ES 0.63) $[39,55,56,60,63-66]$, session volume $>160$ m (ES 0.92) [55, 56, 59, 60, 64], total weekly training volume $>2680 \mathrm{~m}(\mathrm{ES} 0.83)[55,59,60,64]$, and rigid surface (ES 0.69) [39, 56, 61, 64].

should read:

A large ES was obtained for a frequency over twice a week (ES 1.85) [55], and moderate ESs were found for lower loads (ES 0.61) [39, 55-57, 59-65], > 6-week training periods (ES $0.63)[39,55,56,60,63-66]$, session volume $>160 \mathrm{~m}$ (ES $0.92)[55,56,59,60,64]$, total training volume $>2680 \mathrm{~m}$ (ES 0.83) [55, 59, 60, 64], and rigid surface (ES 0.69) [39, $56,61,64]$.

Page 6: Section 3.3, paragraph 3, sentence 3. The following sentence, which read:

Small ESs were also found for a training frequency equal to or fewer than two trainings per week (ES 0.52) [39, 56, 57, 59-66], a total weekly training volume $<2680 \mathrm{~m}$ (ES 0.53 ) $[39,56,57,61-63,65,66]$, and grass surface (ES 0.47) [59, $60,65,66]$.

should read:

Small ESs were also found for a training frequency equal to or fewer than two trainings per week (ES 0.52) $[39,56$, 57, 59-66], a total training volume $<2680 \mathrm{~m}$ (ES 0.53) [39, $56,57,61-63,65,66]$, and grass surface (ES 0.47) [59, 60, $65,66]$.

Page 19: Section 4.1, paragraph 7, sentence 1. The following sentence, which read: 
Another variable that should be considered when designing training programs with RST is volume, both for each session and for the microcycle.

should read:

Another variable that should be considered when designing training programs with RST is volume, both for each session and for the training cycle (i.e. mesocycle).

Page 19: Section 4.1, paragraph 7, sentence 4. The following sentence, which read:

Similarly, when weekly volumes were compared, higher volumes $(>2680 \mathrm{~m})$ produced a moderate effect (ES 0.83) compared with volumes 720-2680 m (ES 0.53), although the effects were statistically significant for both volume per session and weekly volume.

should read:

Similarly, when total training volumes were compared, higher volumes $(>2680 \mathrm{~m})$ produced a moderate effect (ES 0.83) compared with volumes 720-2680 m (ES 0.53), although the effects were statistically significant for both volume per session and total training volume.

Page 20: Section 4.2, paragraph 2, final sentence. The following sentence, which read:

With respect to the training characteristics, the effect was small in all cases and significant with loads $<20 \%$ BM (ES $0.35 ; p=0.02$ ), weekly training frequencies of $\leq 2$ days (ES
$0.35 ; p=0.02)$, for duration $>6$ weeks $(\operatorname{ES~} 0.39 ; p=0.01)$, volumes per session $>160 \mathrm{~m}($ ES $0.53 ; p=0.03)$, and weekly values $>2680 \mathrm{~m}($ ES $0.53 ; p=0.03)$.

should read:

With respect to the training characteristics, the effect was small in all cases and significant with loads $<20 \%$ BM (ES $0.35 ; p=0.02$ ), weekly training frequencies of $\leq 2$ days (ES $0.35 ; p=0.02)$, for duration $>6$ weeks (ES 0.39; $p=0.01$ ), volumes per session $>160 \mathrm{~m}(\operatorname{ES~} 0.53 ; p=0.03)$, and total training values $>2680 \mathrm{~m}($ ES $0.53 ; p=0.03)$.

Page 21: Section 5, paragraph 1, sentence 7. The following sentence, which read:

Finally, with regards to the training characteristics, the intensity (load) is not a determinant of sprint performance improvement, but the recommended volume is $>160 \mathrm{~m}$ per session, and approximately $2680 \mathrm{~m}$ per week, with a training frequency of 2-3 times per week, for at least 6 weeks.

should read:

Finally, with regards to the training characteristics, the intensity (load) is not a determinant of sprint performance improvement, but the recommended volume is $>160 \mathrm{~m}$ per session, and approximately $2680 \mathrm{~m}$ per total training program, with a training frequency of 2-3 times per week, for at least 6 weeks.

The original article has been corrected. 